\title{
Economía de Selva en la Amazonia Suramericana
}

\author{
Jimenez Fajardo, Jarrinson Alejandro \\ Universidad de la Amazonia, Colombia \\ alejandro.jaj25@gmail.com
}

\begin{abstract}
Resumen - La amazonia, es una amplia área geográfica ubicada al sur del continente Americano la cual cuenta con una extensión de aproximada de $5,514,014 \mathrm{Km}^{2}$, en donde desde algunos años atrás ha sido denominada como el pulmón del mundo, debido a los beneficios ecosistemicos, a la multitud y amplia biodiversidad de fauna, flora, especies animales, y comunidades indígenas, los cuales han sido amenazados por la minería y la deforestación en Suramérica, y desde luego otros aspectos de menor fluctuación como la agricultura extensiva, la ganadería y el desarrollo de proyectos de plantas hidroeléctricas. Ahora bien, el objetivo de la presente investigación es describir la economía de la selva en la Amazonia Suramericana desde la perspectiva de la Minería y la Deforestación, sus aportes, impactos y desde luego proponer el turismo como una actividad que permita el fortalecimiento e implementación de estrategias eficientes para la selva y sus pobladores. En este orden de ideas, es importante resaltar que la metodología de investigación empleada es de enfoque cualitativo, se integra al tipo de investigación descriptiva y el desarrollo de herramientas para la recolección de datos fue la entrevista y la revisión bibliográfica en diferentes bases de datos nacionales e internacionales.
\end{abstract}

Palabras clave - Economía; selva; desarrollo; minería; ecosistemas;

\begin{abstract}
The Amazon is a wide geographical area located in the south of the American continent which has an area of approximately $5,514,014 \mathrm{~km}^{2}$, where for some years it has been called the lung of the world, due to the ecosystem benefits, to the multitude and wide biodiversity of fauna, flora,
\end{abstract}

Interconectando Saberes, 2021

ISSN: 2448-8704

(c) EY-NC-ND animal species, and indigenous communities, which have been threatened by mining and deforestation in South America, and of course other aspects of less fluctuation such as extensive agriculture, livestock and the development of projects of hydroelectric plants. Now, the objective of this research is to describe the economy of the jungle in the South American Amazon from the perspective of Mining and Deforestation, its contributions, impacts and of course propose tourism as an activity that allows the strengthening and implementation of efficient strategies for the jungle and its inhabitants. In this order of ideas, it is important to highlight that the research methodology used is of a qualitative approach, it is integrated into the type of descriptive research and the development of tools for data collection was the interview and the bibliographic review in different national databases and international.

Keywords - Economy; jungle; development; mining; ecosystems;

\section{INTRODUCCIÓN}

En la actualidad, la amazonia es una amplia área geográfica ubicada al sur del continente americano la cual cuenta con una extensión de aproximada de 5,514,014 $\mathrm{Km}^{2}$, en donde desde algunos años atrás ha sido denominada el pulmón del mundo, debido a los beneficios ecosistémicos, a la multitud y amplia biodiversidad de fauna, flora, especies animales, y comunidades indígenas. Su Fecha de Recepción: 12 de junio de 2020 Fecha de Aceptación: 09 de noviembre de 2020 Fecha de Publicación: 31 de enero de 2021 
inventario está compuesto por 30,000 tipos de plantas alimenticias, medicinales y demás, cuenta con aproximadamente 2,500 especies de peces, ornamentales y comestibles, seguido de 1,500 tipos de aves migratorias y permanentes, 500 tipos de mamíferos propios de las regiones, 550 tipos de reptiles, y más de 2,500,000 insectos según la OTCA, (2018).

Ahora bien, la amazonia está compuesta por nueve países suramericanos Colombia, Venezuela, Brasil, Perú, ecuador, Bolivia, Guyana, Guayana francesa y Surinam, cada una posee un aspecto importante para la biodiversidad de animales, plantas, aves y especies acuáticas que son vitales para el desarrollo de sus ecosistemas que llevan miles de años en sus hábitats.

Tabla 1, Integración de la Amazonia.

\begin{tabular}{|l|c|c|c|}
\multicolumn{1}{|c|}{ País } & $\begin{array}{c}\text { Área en } \\
\text { metros } \\
\text { cuadrados }\end{array}$ & $\begin{array}{c}\text { Porcentaje } \\
\text { representa } \\
\text { do a nivel } \\
\text { general }\end{array}$ & $\begin{array}{c}\text { Porcentaje } \\
\text { de espacio } \\
\text { a nivel } \\
\text { nacional }\end{array}$ \\
\hline Brasil & $3,600,000 \mathrm{Km}^{2}$ & $65,28 \%$ & $42,5 \%$ \\
\hline Perú & $782,800 \mathrm{Km}^{2}$ & $14,19 \%$ & $60,91 \%$ \\
\hline Colombia & $483,119 \mathrm{Km}^{2}$ & $8,76 \%$ & $42,30 \%$ \\
\hline Venezuela & $178,095 \mathrm{Km}^{2}$ & $3,22 \%$ & $19,43 \%$ \\
\hline Ecuador & $120,000 \mathrm{Km}^{2}$ & $2,17 \%$ & $42,31 \%$ \\
\hline Bolivia & $100,000 \mathrm{Km}^{2}$ & $1,81 \%$ & $10,00 \%$ \\
\hline Guyana & $100,000 \mathrm{Km}^{2}$ & $1,81 \%$ & $45,00 \%$ \\
\hline Surinam & $100,000 \mathrm{Km}^{2}$ & $1,81 \%$ & $62,00 \%$ \\
\hline Guayana & $50,000 \mathrm{Km}^{2}$ & $0,90 \%$ & $70,00 \%$ \\
francesa & $5,514,014$ & $100 \%$ & \\
\hline Total & $\mathbf{K m}{ }^{2}$ & &
\end{tabular}

Fuente: Reconstrucción propia, (2020).

En este orden de ideas, se puede mencionar que la riqueza de la selva amazónica radica en su biodiversidad en especies y en las comunidades indígenas que habitan desde generaciones en la selva tropical las cuales emplean y desarrollan métodos rudimentarios para su existencia en la agricultura extensiva y la ganadería de porcinos, aves y otras especies menores, y desde luego lo que el ambiente les permita cosechar, permitiendo así su existencia sin destruir y deforestar grandes cantidades de espacios, admitiendo así generar y concebir beneficios tales como.

- El $25 \%$ de los fármacos existentes en el mundo existen gracias a las plantas descubiertas en la selva amazónica.

- Permite la estabilización del clima a nivel global mediante la absorción del dióxido de carbono.

- Es vital para mantener los ciclos de agua y evitar sequias a nivel global.

- Cuenta con ricos suelos.

- Es generadora y productora de múltiples comodities

- Es rica en petróleo, oro, diamantes, esmeraldas y coltan, entre otros minerales.

- Amplia biodiversidad en fauna, flora, peces y especies de animales.

Por otro lado, la selva amazónica así como manifiesta sus beneficios y ventajas ecosistemicas, presenta problemas y situaciones que la están devastando poco a poco, entre dichos factores se pueden evidenciar la deforestación, la minería y la contaminación de la selva, el desarrollo y la construcción de hidroeléctricas, provocando con esto el desplazamiento de comunidades indígenas y finalmente los grupos armados y las mafias toman más fuerza, (es importante resaltar que no hablaremos en esta investigación de los dos últimos factores). 
Consecuentemente se puede definir que la presente investigación tiene como objetivo describir la economía de la selva en la Amazonia Suramericana desde el enfoque de la Minería y la Deforestación, sus aportes, impactos y desde luego proponer el turismo como una acción que permita el fortalecimiento e implementación de estrategias eficientes para la selva y sus pobladores. De igual manera se puede mencionar que la metodología de investigación empleada es de enfoque cualitativo, se integra al tipo de investigación descriptiva y el desarrollo de herramientas para la recolección de datos fue la entrevista semiestructurada y la revisión bibliográfica en diferentes bases de datos nacionales e internacionales sobre temas de biodiversidad, minería, desplazamientos de especies debido a la deforestación y finalmente se aborda el aspecto legal, las normas y políticas que contribuyen a controlar dichas actividades.

\section{Metodología}

La amazonia es un mundo de información vital para la vida de diversas especies, comunidades indígenas, campesinos y familias que buscan mejorar su calidad de vida por medio de la agricultura, la ganadería, minería, y demás actividades que le permitan generar ingresos para su sostenibilidad, fomentando así el $40 \%$ de la informalidad y el desarrollo de actividades ilegales en los nueve países de Suramérica que componen la amazonia.

De esta manera nace el objetivo de la presente investigación, el cual se enfoca en describir la economía de la selva amazónica en los nueve países que la estructuran (Colombia, Venezuela, Brasil, Perú, ecuador, Bolivia, Guyana, Guayana francesa y Surinam), de igual manera detallar sus impactos y proponer el turismo como una posible solución que busque la eficiencia sostenible y el fortalecimiento en actividades legales, responsables y sustentables para el mejoramiento de la calidad de vida de la población.

Ahora bien, el enfoque empleado para desarrollar la presente investigación es interpretado como cualitativo, debido a que Sampieri, Baptista y Hernández (2014, p.08) lo conceptualizan en:

"Un conjunto de métodos de recolección de datos no estandarizados ni predeterminados completamente. Tal recolección consiste en obtener las perspectivas y puntos de vista de los participantes (sus emociones, prioridades, experiencias, significados y otros aspectos más bien subjetivos). También resultan de interés las interacciones entre individuos, grupos y colectividades".

En este orden de ideas, y luego de haber detallado el enfoque de investigación empleado, se debe mencionar que el tipo de investigación desarrollado en el presente estudio es de carácter descriptivo, en donde "se busca especificar las propiedades y las características de un fenómeno específico que se someta a un análisis. Es decir, únicamente pretenden medir o recoger información de manera independiente o conjunta sobre los conceptos o las variables a las que se refieren, esto es, su objetivo no es indicar cómo se relacionan éstas" (Baptista, Sampieri y Hernández (2014, p.92). 
Ahora bien, frente a los métodos de recolección y obtención de datos se puede destacar que se emplearon dos procesos, inicialmente la entrevista estructura a profesionales experto del tema, y la revisión bibliográfica en diferentes bases de datos nacionales e internacionales (E-libro, S-cielo, Biblioteca virtual de la Universidad Nacional de Colombia), en donde se reafirma lo mencionado anteriormente en el enfoque investigativo, ya que Baptista, Sampieri y Hernández (2014, p.09) detallan que este enfoque "utiliza técnicas para recolectar datos, como la observación no estructurada, entrevistas semiestructuradas, revisión de documentos, discusión en grupo, evaluación de experiencias personales, registro de historias de vida, e interacción e introspección con grupos o comunidades".

De esta manera, se describen el enfoque y el tipo de investigación empleados en la presente investigación, al igual que se establecen las herramientas y elementos de recolección de información. A continuación, se detalla de manera minuciosa las fases del estudio:

- Fase I Idea: Inicialmente la idea nace debido a la necesidad de identificar cual es la economía que genera la población que habita la selva, seguido de ello, identificar los aspectos que generan algún tipo de impacto en el aspecto social, económico y ambiental.

- Fase II Planteamiento del problema: ¿Cómo incide la economía de la selva en la amazonia suramericana?
- Fase III Inmersión en el campo: La amazonia integrada en Brasil, Colombia, Venezuela, Perú, ecuador, Bolivia, Guyana, Surinam, Guayana francesa.

- Fase IV Elección de instrumentos o herramientas: Entrevista semiestructurada, compuesta por cinco preguntas específicas, revisión bibliográfica.

- Fase V: Definición de la muestra inicial del estudio: La muestra del estudio frente a la recolección de datos son cuatro profesionales, doctores en temas de desarrollo sostenible y medio ambiente.

- Fase VI: Recolección de los datos: Inicialmente para la recolección de datos primero se empleó la aplicación de cuatro entrevistas a profesionales y doctores en temas de desarrollo sostenible y medio ambiente, y como herramienta de recolección de datos secundaria se desarrolló el método de revisión bibliográfica en diferentes bases de datos nacionales e internacionales.

- Fase VII: Análisis de datos: De acuerdo a la obtención de datos, estos se establecieron en una matriz de análisis y diagnóstico de factores críticos de riesgo.

- Fase VIII Interpretación de datos: Se resaltaron los factores de mayor incidencia e impacto que provoca la economía de la selva en la actualidad.

- Fase IX Conclusiones: Se plantean acciones estratégicas que permiten la sostenibilidad y el mejoramiento de la calidad de vida de la población en la selva. 


\section{Resultados}

\section{La deforestación en la amazonia}

La deforestación en términos sencillos, "es la extinción, exterminio o tala de árboles" lo cual provoca a su vez diferentes impactos ambientales y culturales en la selva amazónica (Juste, 2019; Jara, 2015). Ahora bien, la deforestación en primer lugar es un fenómeno de destrucción, pero a su vez es la forma con la cual miles de familias ubicadas en la amazonia general reciben su alimento, bien sea por la tala, transporte, venta o comercialización de dicha madera, ya que es el producto final de árboles que llevan cientos de años en las cuencas (Torracchi, 2015; Monjardin, Pacheco, Plata, y Corrales, 2017; Miranda, 2013).

Es importante resaltar que, la deforestación no solamente se lleva a cabo bajo la mano del hombre, si no que existe una forma natural donde la naturaleza de forma inexplicable realiza dicho proceso, bien sea con el crecimiento o desborde de cuencas o ríos, o por derrumbes de tierra que sepultan una capa vegetal de la selva (Urquiza y Burga, 2016; García, 2008; Chadid, 2014).

En este orden de ideas, se puede detallar que la deforestación en la amazonia es producida inicialmente para la sobrevivencia de las familias con el objetivo de sembrar y cosechar sus productos agrícolas, seguido de esto, se puede detallar que las mafias y el narcotráfico también generan un alto impacto, pero como se mencionó inicialmente, en la presente investigación no se hablara de mafia, ni de narcotráfico, ya que el objeto de estudio es describir y caracterizar la economía que existe en la selva de la amazonia; continuando con los factores que provocan la deforestación, se puede evidenciar que las invasiones (apropiarse de un terreno que es privado o es del Estado) generan impactos iguales en la selva, ya que las invasiones generan contaminación, deforestación, desplazamiento de animales, daño al medio ambiente y en general un daño irreversible a la selva.

Actualmente los impactos de la deforestación en la amazonia suramericana son bastante amplios, ya que la deforestación aporta de manera negativa al calentamiento global del planeta, provocando con esto altas temperaturas, pocas Iluvias, sequia, la muerte de animales como el ganado bobino, porcino, caprino, entre otros, y finalmente la devastación de la biodiversidad en diferentes partes del mundo (Gutiérrez, 2016; Suqui y Merecí, 2014). ¿Por qué es tan importante la amazonia suramericana? La amazonia suramericana es caracterizada por su amplia cantidad de árboles, insectos, mamíferos, diversas especies y sobre todo por su cantidad de agua dulce denominando de esta manera que es el pulmón del mundo. Australia, hasta hace poco era un importante aportante para la reducción de las emisiones de carbono, y el calentamiento global, pero todo quedo hecho cenizas. Ahora bien, la selva suramericana es vital para el mundo, porque hasta el momento es la cuenca selvática más grande del planeta (Monjardin, et al, 2017; Guerra, 2016; Milán, 2017). 
En este orden ideas, y luego de haber establecido el aporte del sector forestal a la economía de los países que integran la amazonia, se describirán a continuación los impactos según la $F A O$ y MAGBMA, (2016).

- En Brasil, la deforestación avanza a pasos de gigante, ya que en los últimos diez años la pérdida de su capa vegetal oscila entre los $300,000 \mathrm{~km}^{2}$ evidenciando con esto que prácticamente el $13 \%$ de su selva tiene daños irreversibles debido a la minería, y a prácticas ilegales como la siembra de coca, amapola y otros tipos de cultivos.

- En Bolivia, la pérdida del bosque y la selva amazónica ha sido aproximadamente de $10,000 \mathrm{~km}^{2}$ entre los últimos años. Algunas de las causas que han provocado la deforestación han nacido en la ganadería y la agricultura en escala.

- En Colombia, el panorama es diferente. La deforestación avanza a pasos lentos pero irreparables para la biodiversidad y las comunidades indígenas; de acuerdo a diferentes boletines del IDEAM se puede aclarar que la perdida de capa vegetal de la selva amazónica en Colombia es prácticamente de $8,000 \mathrm{~km}^{2}$ en donde la selva y las zonas boscosas han sido invadidas por personas que no tienen vivienda, apropiándose y construyendo sus casas en estos lugares. No obstante, la minería y el crecimiento de la frontera agrícola han generado altos porcentajes de deforestación.

- En Perú, el aspecto no mejora siendo el cuarto país con mayor nivel de deforestación en Suramérica, ya que durante los últimos años el
$10 \%$ de su selva se ha perdido a raíz de la agricultura principalmente por la siembra de cacao, y palma de aceite.

- En Surinam, Guyane y Guayana francesa, la selva ha recibido el peor impacto, ya que por la necesidad de incrementar y hacer que crezca la economía han construido hidroeléctricas para la generación de energía eléctrica, provocando la devastación y la perdida de la capa vegetal en el $12 \%$ del territorio.

- En Venezuela, es el único país en donde su porcentaje es de deforestación es del 3,3\%, y es producido debido a la minería ilegal y a la extracción del petróleo.

- En Ecuador, la deforestación se posiciona en segundo lugar, provocando la pérdida del 10,7\% de capa vegetal debido a la extracción de materias primas y minerales de la tierra.

Ahora bien, la amazonia alberga una amplia cantidad de minerales, elementos y materias primas e insumos que son empleados por las empresas, pero estos deben ser explotados bajo buenas prácticas manufactureras, ya que un mal uso de la tierra podría generar grandes impactos (Boton, 2013; Martínez, 2019).

Por ende, los diferentes países que componen la amazonia han creado leyes, normas e instituciones para desarrollar un buen manejo a los recursos de la selva, inicialmente para su protección y los planes de mitigación de la deforestación, permitiendo así tener una compensación ambiental, tanto económica, como de resembrar pequeños árboles. 
Tabla 2, Normatividad y Marco Legal de protección para la Amazonia.

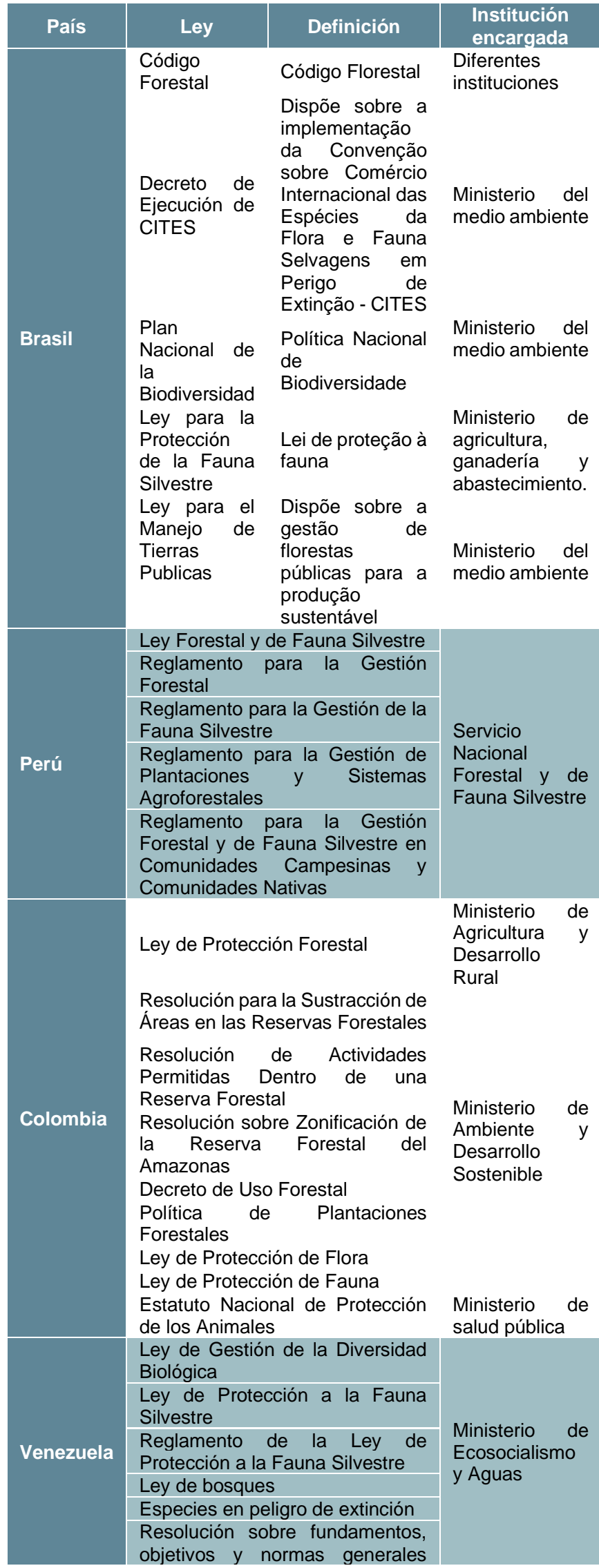

\begin{tabular}{|c|c|c|}
\hline & $\begin{array}{l}\text { para la ejecución del programa } \\
\text { del manejo integral comunitario } \\
\text { del bosque }\end{array}$ & \\
\hline Ecuador & $\begin{array}{l}\text { Ley Forestal y de Áreas Naturales } \\
\text { Protegidas y sus reglamentos }\end{array}$ & $\begin{array}{l}\text { Ministerio del } \\
\text { Medio ambiente }\end{array}$ \\
\hline \multirow{3}{*}{ Bolivia } & Ley forestal & \multirow{3}{*}{$\begin{array}{l}\text { Ministerio del } \\
\text { Medio ambiente } \\
\text { y agua }\end{array}$} \\
\hline & $\begin{array}{l}\text { Ley Que Aprueba y } \begin{array}{l}\text { Ratifica el } \\
\text { Convenio Sobre } \\
\text { Biológica de NNUU. }\end{array} \\
\end{array}$ & \\
\hline & $\begin{array}{l}\text { Reglamento general de la ley } \\
\text { forestal }\end{array}$ & \\
\hline \multirow[b]{2}{*}{ Guyana } & $\begin{array}{l}\text { Ley forestal } \\
\text { Ley de la comisión forestal } \\
\text { Política forestal nacional }\end{array}$ & $\begin{array}{l}\text { Comisión } \\
\text { Forestal }\end{array}$ \\
\hline & $\begin{array}{l}\text { Plan forestal Nacional } \\
\text { Estrategia Nacional y plan de } \\
\text { acción de biodiversidad }\end{array}$ & $\begin{array}{l}\text { Agencia de } \\
\text { protección } \\
\text { medioambiental }\end{array}$ \\
\hline \multirow[b]{2}{*}{ Suriname } & Ley de gestión forestal & \multirow[b]{2}{*}{$\begin{array}{l}\text { Ministerio de } \\
\text { Planificación } \\
\text { Espacial, } \\
\text { Territorial, y } \\
\text { Manejo } \\
\text { Bosques }\end{array}$} \\
\hline & $\begin{array}{l}\text { Ley de protección de fauna } \\
\text { silvestre }\end{array}$ & \\
\hline
\end{tabular}

Fuente: Reconstrucción propia, (2020).

Ahora bien, es importante mencionar que la amazonia integrada por los nueve países suramericanos aporta al Producto Interno Bruto por medio del sector forestal el $0,9 \%$ un porcentaje estable durante diferentes años consecutivos, relativamente se puede aclarar que es un valor significativo, en donde este podría llegar hacer mayor si la ilegalidad y la informalidad no existiera (UPRA, 2015; FAO, 2015; IDEAM, 2013). No obstante, es importante resaltar que el sector forestal genera el $0,4 \%$ de empleos directos e indirectos en los nueve países (ONF Andina, 2018; Vera, 2015).

\section{La minería en la Amazonia.}

Inicialmente se puede interpretar que la minería es una actividad económica del sector primario que se dedica a explotar la tierra para la extracción de minerales, actividad de la cual la amazonia es el mayor triunfo de las multinacionales debido a su amplia diversidad de minerales tales como el oro, coltan, piedras preciosas, diamantes, entre otros. 
Ahora bien, es importante resaltar que el aporte de la minería al Producto Interno Bruto de la amazonia es diferente de acuerdo al país, en el caso de Colombia la minería aporta el $2 \%$ al PIB, aparte de ello genera 350,000 empleos directos y 1.000 .000 de empleos indirectos, produciendo regalías e impuestos de US\$198 millones para el año 2018 (Portafolio, 2019; 2019). Es importante resaltar, que en Colombia los aportes de la minería se encuentran enfocados a favorecer los sectores de la educación, salud, construcciones de puentes, acueductos, redes eléctricas y otros servicios públicos que presta el estado colombiano (Agencia Nacional de minería, 2014; FEDESARROLLO, 2008).

En el caso de Brasil, la minería aporta el $10 \%$ al Producto Interno Bruto del país, aparte de ello, es denominado con la sexta nación minera más grande del mundo aportando oro, níquel, aluminio, bauxita, cobre, fosfatos, hierro, niobio, esmeraldas, diamantes, potasio, entre otros, materiales extraído de la amazonia brasileña, bajo estándares y normas enfocadas a las buenas prácticas de manufactura. Seguido de ello, se puede resaltar que produce 749.000 empleos directos y más de 3.000.000 de empleos indirectos (CEPAL, 2011; CEPAL, 2018).

Por otro lado, en el caso del Perú el aporte de la minería al PIB es del 10\%, generando con esto mayores divisas e ingresos fiscales por impuestos, la creación de más de 400,000 empleos directos y 700,000 indirectos. Es importante resaltar que la minería en Perú produce plata, cobre, zinc, estaño, plomo y oro, teniendo como resultado el pago de impuestos en aproximadamente 9,000 millones de soles, o sea, 2.621.995.620 de dólares americanos.

No obstante, en el caso de Ecuador se puede observar que vive el inicio de un crecimiento bastante importante debido al hallazgo de nuevos minerales en la selva amazónica, detallando de esta manera un aumento en el sector minero del país que para el 2017 su aporte era de 1,7\% en producto interno bruto, y del $4 \%$ proyectándolo hacia el 2021. Ocasionando con esto 30,000 empleos directos e indirectos en el país, y más 800 millones de dólares en el pago de impuestos, recursos que se enfocaran en la inversión social del país (Ministerio de economía y finanzas Ecuador, 2018; El comercio, 2019).

En Bolivia, el Ministerio de Minería detalla en su informe de crecimiento y expansión económica el reporte por impuesto de 235 millones de dólares para el año 2017 de las diferentes empresas situadas en el país, de donde extraen zinc, plata, plomo, estaño, cobre y oro. Generando de esta manera 136,848 empleos directos y más de 821.000 empleos indirectos, diciendo con esto que la minería es el segundo renglón más importante de la economía en el país (Ministerio de Minería, 2018).

En este orden de ideas, Venezuela no se queda atrás en el sector minero, todo lo contrario, Venezuela posee altas cuotas de reservas mundiales en petróleo, oro, hierro, la bauxita, 
carbón, arcilla, arena, caliza, feldespato y sílice, entre otros. Son tan altas las cantidades de estos minerales que prácticamente entre el $10 \%$ y el $23 \%$ de estos minerales los posee Venezuela. De acuerdo con Jiménez, (2015), Venezuela posee 10,000 millones de toneladas en reservas de carbón y más 6,000 millones de toneladas en bauxita, es importante detallar que de la bauxita se produce el aluminio. Conforme con lo mencionado, la minería solo aporta el $1,07 \%$ al PIB del país, debido a la falta de aliados estratégicos y compradores potenciales. Venezuela cuenta con aproximadamente 7,000 millones de toneladas de oro, y a nivel mundial ocupa el puesto número 16 de los países más ricos del mundo en riqueza mineral.

\section{Matriz de análisis}

De acuerdo con los diferentes temas relacionados anteriormente, se tomaron fuentes primarias. A continuación, se relacionan cuatro entrevistas a cuatro doctores expertos en temas de desarrollo sostenibles y medio ambiente que han estudiado e investigado la selva amazónica, el instrumento está compuesto por cinco preguntas en donde se integran diferentes factores para la economía de selva.

Tabla 3, Tabulación de datos recolectados.

\begin{tabular}{|c|c|c|c|c|}
\hline $\begin{array}{l}\text { Pregunta/ } \\
\text { Actores }\end{array}$ & Individuo & $\overline{\text { Individuo }}$ & Individuo & $\begin{array}{c}\text { Individuo } \\
\text { IV }\end{array}$ \\
\hline $\begin{array}{l}\text { ¿Qué } \\
\text { opina de } \\
\text { la } \\
\text { deforestac } \\
\text { ión en la } \\
\text { amazonia } \\
?\end{array}$ & $\begin{array}{l}\text { La } \\
\text { actividad } \\
\text { de la } \\
\text { deforesta } \\
\text { ción en la } \\
\text { selva } \\
\text { amazónic } \\
\text { a bien } \\
\text { sea en } \\
\text { Colombia, } \\
\text { Brasil, }\end{array}$ & $\begin{array}{l}\text { La tala de } \\
\text { árboles es } \\
\text { una } \\
\text { actividad } \\
\text { del sector } \\
\text { primario } \\
\text { que les } \\
\text { permite a } \\
\text { muchas } \\
\text { familias } \\
\text { campesina }\end{array}$ & $\begin{array}{l}\text { La } \\
\text { deforestac } \\
\text { ión es uno } \\
\text { de los } \\
\text { mayores } \\
\text { fenómeno } \\
\text { s que } \\
\text { existen en } \\
\text { la selva y } \\
\text { más aún } \\
\text { en las }\end{array}$ & $\begin{array}{l}\text { El talar } \\
\text { árboles } \\
\text { para } \\
\text { muchas } \\
\text { familias en } \\
\text { el sustento } \\
\text { diario, pero } \\
\text { esto no es } \\
\text { justificació } \\
\text { n para } \\
\text { destruir y }\end{array}$ \\
\hline
\end{tabular}

\begin{tabular}{|c|c|c|c|c|}
\hline $\begin{array}{l}\text { Pregunta/ } \\
\text { Actores }\end{array}$ & Individuo & Individuo & $\begin{array}{c}\text { Individuo } \\
\text { III }\end{array}$ & $\begin{array}{l}\text { Individuo } \\
\text { IV }\end{array}$ \\
\hline & $\begin{array}{l}\text { Perú o } \\
\text { cualquier } \\
\text { país } \\
\text { surameric } \\
\text { ano es } \\
\text { una } \\
\text { acción } \\
\text { que } \\
\text { devasta } \\
\text { la vida, la } \\
\text { biodiversi } \\
\text { dad y } \\
\text { perjudica } \\
\text { la selva, } \\
\text { los } \\
\text { indígenas } \\
\text { y en } \\
\text { general } \\
\text { provoca } \\
\text { la } \\
\text { destrucci } \\
\text { ón. }\end{array}$ & $\begin{array}{l}\text { s adquirir } \\
\text { dinero } \\
\text { para } \\
\text { comprar } \\
\text { sus } \\
\text { alimentos. } \\
\text { Además, } \\
\text { la tala de } \\
\text { árboles no } \\
\text { perjudica } \\
\text { la selva, } \\
\text { siempre y } \\
\text { cuando se } \\
\text { re- } \\
\text { siembren } \\
\text { los árboles } \\
\text { talados. }\end{array}$ & $\begin{array}{l}\text { cuencas } \\
\text { amazónic } \\
\text { as, ya que } \\
\text { sin la } \\
\text { selva no } \\
\text { hay vida, } \\
\text { no hay } \\
\text { aire, no } \\
\text { hay } \\
\text { biodiversid } \\
\text { ad. }\end{array}$ & $\begin{array}{l}\text { deplorar la } \\
\text { selva, } \\
\text { además no } \\
\text { solo los } \\
\text { campesino } \\
\text { s talan, si } \\
\text { no grandes } \\
\text { compañías } \\
\text { internacion } \\
\text { ales que } \\
\text { no les } \\
\text { importan } \\
\text { los } \\
\text { resultados } \\
\text { que la } \\
\text { deforestaci } \\
\text { ón } \\
\text { provoca. }\end{array}$ \\
\hline $\begin{array}{l}\text { ¿Cuál es } \\
\text { su } \\
\text { percepció } \\
\mathrm{n} \text { de la } \\
\text { minería en } \\
\text { la selva? }\end{array}$ & $\begin{array}{l}\text { En mi } \\
\text { opinión } \\
\text { es } \\
\text { totalment } \\
\text { e pésima, } \\
\text { su aporte } \\
\text { al PIB } \\
\text { (Producto } \\
\text { Interno } \\
\text { Bruto) es } \\
\text { mediocre } \\
\text { y los } \\
\text { impactos } \\
\text { son } \\
\text { irreversibl } \\
\text { es. }\end{array}$ & $\begin{array}{l}\text { La minería } \\
\text { desde un } \\
\text { uso y } \\
\text { manejo } \\
\text { adecuado } \\
\text { genera } \\
\text { empleos, } \\
\text { desarrollo, } \\
\text { competitivi } \\
\text { dad, } \\
\text { regalías e } \\
\text { ingresos a } \\
\text { las } \\
\text { diferentes } \\
\text { regiones } \\
\text { de los } \\
\text { países que } \\
\text { conforman } \\
\text { la } \\
\text { amazonia. }\end{array}$ & $\begin{array}{l}\text { La minería } \\
\text { al igual } \\
\text { que la } \\
\text { deforestac } \\
\text { ión } \\
\text { provoca } \\
\text { diferentes } \\
\text { tipos de } \\
\text { impactos } \\
\text { ambiental } \\
\text { es, entre } \\
\text { estos la } \\
\text { contamina } \\
\text { ción en el } \\
\text { agua, en } \\
\text { suelos y } \\
\text { hasta en } \\
\text { el aire, } \\
\text { generando } \\
\text { el } \\
\text { exterminio } \\
\text { de } \\
\text { animales, } \\
\text { fauna y } \\
\text { flora. }\end{array}$ & $\begin{array}{l}\text { La minería } \\
\text { desde un } \\
\text { aspecto } \\
\text { sostenible, } \\
\text { y con el } \\
\text { desarrollo } \\
\text { de buenas } \\
\text { prácticas } \\
\text { manufactur } \\
\text { eras } \\
\text { pueden } \\
\text { generan } \\
\text { utilidades } \\
\text { importante } \\
\text { s para el } \\
\text { país, el } \\
\text { problema } \\
\text { radica es } \\
\text { en la } \\
\text { minería } \\
\text { ilegal. }\end{array}$ \\
\hline $\begin{array}{l}\text { ¿Cree que } \\
\text { las } \\
\text { hidroeléctr } \\
\text { icas } \\
\text { generaría } \\
\text { n } \\
\text { desarrollo } \\
\text { para la } \\
\text { selva } \\
\text { amazónic } \\
\text { a de } \\
\text { Suraméric } \\
\text { a? }\end{array}$ & $\begin{array}{l}\text { Sí, pero a } \\
\text { ¿qué } \\
\text { impacto? } \\
\text { Se secan } \\
\text { ríos, } \\
\text { mueren } \\
\text { peces, los } \\
\text { animales } \\
\text { son } \\
\text { desplaza } \\
\text { dos, la } \\
\text { fauna y la } \\
\text { flora son } \\
\text { destruida } \\
\text { s. }\end{array}$ & $\begin{array}{l}\text { Si, } \\
\text { generan } \\
\text { empleo, } \\
\text { desarrollo, } \\
\text { competitivi } \\
\text { dad, } \\
\text { mayor } \\
\text { desempeñ } \\
\text { o regional. }\end{array}$ & $\begin{array}{l}\text { Sí, pero } \\
\text { se deben } \\
\text { manejar } \\
\text { muy } \\
\text { buenas } \\
\text { prácticas } \\
\text { que no } \\
\text { contamine } \\
\text { n el } \\
\text { ambiente } \\
\text { y la selva, } \\
\text { permitiend } \\
\text { o que los } \\
\text { animales } \\
\text { mantenga } \\
n \text { su vida. }\end{array}$ & $\begin{array}{l}\text { No, debido } \\
\text { a que } \\
\text { provocan } \\
\text { diferentes } \\
\text { impactos } \\
\text { ambientale } \\
\text { s y a largo } \\
\text { plazo } \\
\text { generan } \\
\text { desventaja } \\
\text { s a las } \\
\text { regiones. }\end{array}$ \\
\hline $\begin{array}{l}\text { ¿Conoce } \\
\text { los } \\
\text { canales } \\
\text { de acceso } \\
\text { a la selva } \\
\text { amazónic } \\
\text { a? }\end{array}$ & $\begin{array}{l}\text { Si claro, } \\
\text { he } \\
\text { desarrolla } \\
\text { do más } \\
\text { de } 50 \\
\text { expedicio } \\
\text { nes en la } \\
\text { búsqueda } \\
\text { de }\end{array}$ & $\begin{array}{l}\text { Mi } \\
\text { experienci } \\
\text { a en } \\
\text { campo es } \\
\text { bastante } \\
\text { amplia y } \\
\text { normalme } \\
\text { nte nos } \\
\text { trasladamo }\end{array}$ & $\begin{array}{l}\text { A la selva } \\
\text { se puede } \\
\text { ingresar } \\
\text { de dos } \\
\text { maneras } \\
\text { seguras, } \\
\text { vía fluvial } \\
\text { por los } \\
\text { ríos o vía }\end{array}$ & $\begin{array}{l}\text { Sí, claro. } \\
\text { La vía } \\
\text { fluvial es la } \\
\text { más } \\
\text { segura. En } \\
\text { algunos } \\
\text { lugares vía } \\
\text { aérea y vía } \\
\text { terrestre }\end{array}$ \\
\hline
\end{tabular}




\begin{tabular}{|c|c|c|c|c|}
\hline $\begin{array}{c}\text { Pregunta/ } \\
\text { Actores }\end{array}$ & $\begin{array}{c}\text { Individuo } \\
\text { I }\end{array}$ & $\begin{array}{c}\text { Individuo } \\
\text { II }\end{array}$ & $\begin{array}{c}\text { Individuo } \\
\text { III }\end{array}$ & $\begin{array}{c}\text { Individuo } \\
\text { IV }\end{array}$ \\
\hline & $\begin{array}{l}\text { nuevas } \\
\text { especies, } \\
\text { fauna y } \\
\text { flora. } \\
\text { Solo se } \\
\text { ingresa } \\
\text { por medio } \\
\text { del rio y } \\
\text { en } \\
\text { algunos } \\
\text { lugares } \\
\text { en } \\
\text { avioneta. }\end{array}$ & $\begin{array}{l}\text { s en } \\
\text { canoas en } \\
\text { la vía } \\
\text { fluvial. }\end{array}$ & $\begin{array}{l}\text { terrestre } \\
\text { por medio } \\
\text { de guías } \\
\text { indígenas } \\
\text { que } \\
\text { conocen } \\
\text { sus } \\
\text { territorios. }\end{array}$ & $\begin{array}{l}\text { por medio } \\
\text { de los } \\
\text { indígenas y } \\
\text { vías } \\
\text { terciarios } \\
\text { creadas } \\
\text { ilegalmente }\end{array}$ \\
\hline $\begin{array}{l}\text { ¿Qué } \\
\text { opina } \\
\text { sobre el } \\
\text { desarrollo } \\
\text { del sector } \\
\text { primario } \\
\text { en la } \\
\text { amazonia } \\
?\end{array}$ & $\begin{array}{l}\text { Es } \\
\text { totalment } \\
\text { e } \\
\text { extensiva } \\
\text { y poco } \\
\text { productiv } \\
\text { a en la } \\
\text { selva } \\
\text { amazónic } \\
\text { a. }\end{array}$ & $\begin{array}{l}\text { Para la } \\
\text { amazonia, } \\
\text { la } \\
\text { agricultura } \\
\text { y el sector } \\
\text { pecuario } \\
\text { son } \\
\text { extensivo } \\
\text { debido a la } \\
\text { falta de } \\
\text { recursos } \\
\text { monetarios } \\
\text {. }\end{array}$ & $\begin{array}{l}\text { Es } \\
\text { extensiva } \\
\text { prácticam } \\
\text { ente para } \\
\text { el } 99 \% \text { de } \\
\text { la } \\
\text { población } \\
\text { que vive } \\
\text { en la } \\
\text { selva. }\end{array}$ & $\begin{array}{l}\text { La selva } \\
\text { tiene una } \\
\text { amplia } \\
\text { capacidad } \\
\text { de producir } \\
\text { y generar } \\
\text { diferentes } \\
\text { tipos de } \\
\text { comodities, } \\
\text { que hoy en } \\
\text { día son } \\
\text { muy } \\
\text { importante } \\
\text { s ya que } \\
\text { relacionan } \\
\text { diferentes } \\
\text { minerales } \\
\text { como el } \\
\text { oro y el } \\
\text { coltan. }\end{array}$ \\
\hline $\begin{array}{l}\text { Informació } \\
n \\
\text { emergente } \\
\text { i }\end{array}$ & $\begin{array}{l}\text { Ingresos } \\
\text { de la } \\
\text { minería al } \\
\text { PIB. }\end{array}$ & & & $\begin{array}{l}\text { Minería } \\
\text { llegal. }\end{array}$ \\
\hline
\end{tabular}

Fuente: Reconstrucción propia, (2020).

\section{El turismo como propuesta de sostenibilidad}

El turismo, es una actividad económica en constante cambio y flexibilidad debido a las necesidades del mercado y a las diferentes tendencias existentes en la sociedad. Ahora bien, el turismo se puede definir como una actividad o afición que consiste en viajar por placer, descanso o relajación. Esta definición, ha evolucionado a medida que ha pasado el tiempo, ya que inicialmente hace algunas décadas atrás los únicos que viajaban eran los pensionados o personas de la tercera edad, luego de ello, se dio inicio al turismo masificado, en donde cientos de personas se aglomeraban en un playa, piscina o lugar que llamara la atención.

Actualmente, el turismo ha evolucionado y se ha clasificado de acuerdo con el tipo de viaje de los turistas, entre estos podemos encontrar el turismo de aventura, el ecoturismo, etnoturismo, agroturismo comunitario, turismo rural, cicloturismo, turismo de salud o de bienestar, turismos de negocio, gastronómico, urbano, marítimo o de crucero entre otros; generando con esto que los turistas se han los que tomen la decisión de como desean pasar sus vacaciones.

Es aquí en donde el turismo de aventura, etnoturismo, ecoturismo y el agroturismo comunitario se convierten en la mayor riqueza y actividad económica a desarrollar en la amazonia compuesta por sus nueve países, debido a que no genera ningún tipo de amenaza de deforestación, o algún riesgo para la biodiversidad en la selva.

No obstante, el turismo es una actividad económica que genera importante utilidades para el sector terciario y para el Producto Interno Bruto de los países, de acuerdo a la Organización Mundial del Turismos (2017), el turismo aporta al PIB mundial $10,4 \%$ generando con ello que una de cada diez personas en el mundo trabaje, labore o desarrolle alguna actividad en el sector del turismo, comprendiendo restaurantes, hoteles, transporte, guías turísticos, entre otros (Mogrovejo, et al, 2019; Ortiz, Et al, 2019). 
Es así como resulta siendo el turismo, una actividad vital para la generación de utilidades en la amazonia, inicialmente por la conservación y el cuidado de la selva. En el caso de Colombia, el turismo aporto el 6,9\% al PIB para el año 2018. En el caso de Brasil para el año 2016 el turismo aporto al PIB el 8,5\%, generando de esta manera 152,000 millones de dólares (Informe de PIB, 2019). Ahora bien, en el caso de Perú el aporte al PIB por parte del turismo es de 20,838 millones de dólares, o sea, un aporte de 9,8\% para el año 2018 (Cámara Nacional del Turismo del Perú, 2018). Para el caso de Ecuador, el turismo aporto el 5,51\% al PIB, generando 24,720 millones de dólares para el año 2018 (Ministerio de turismo, 2018). En el caso de Bolivia, el turismo aporto para el 2018 1,024 millones de dólares, representando el $4,57 \%$ del aporte al PIB (Bolivia Emprende, 2018). Frente al caso de Venezuela, el turismo es una actividad económica que está en decreciente debido a diferentes factores sociales y políticos, pero aun así continúa generando utilidades importantes, para el año 2013 aporto aproximadamente 15,283 millones de dólares al PIB y para el año 2018 el aporte fue de 6,392 millones de dólares (Bautista, 2019).

\section{CONCLUSIONES}

Finalmente, se puede mencionar que la deforestación en la amazonia suramericana genera diferentes impactos sociales, económicos, culturales y sobre todo ambientales. Sociales en el sentido de que genera protestas, marchas, y diferentes tipos de movilizaciones de pequeños grupos de personas que rechazan este tipo de actividades en la amazonia. En segundo lugar se conciben Impactos económicos debido a que de alguna $u$ otra forma se afecta el Producto Interno Bruto de los países, tal y como se observó anteriormente. En tercer lugar, se crean impactos culturales, debido a que las etnias indígenas que habitan la selva se deben desplazar para poder ocultarse o salir a luz pública e iniciar nuevos procesos de adaptación a la sociedad y finalmente el impacto ambiental que genera la deforestación en la selva, del cual se mencionó anteriormente.

Ahora bien, la minería es una actividad económica que genera diferentes impactos para la economía, debido a que sus aportes se enfocan en la educación, salud, y diferentes tipos de inversiones sociales que construyen al desarrollo de la sociedad, seguido de ello, genera impactos ambientales como la deforestación y sus efectos en la selva.

En conclusión, el turismo es una actividad económica que les permite a los países amazónicos generar desarrollo sin provocar impactos que comprometan el ambiente y sin poner en riesgo la biodiversidad y sus componentes, permitiendo así conservar y resguardar su riqueza natural y paisajística.

\section{REFERENCIAS}

Agencia Nacional de minería, (2014). Los beneficios de la minería. Recuperado en https://www.anm.gov.co/portalninos/benefici os-mineria

Baptista, P. Sampieri, R. y Fernández, C. (2014). Metodología de la investigación. Sexta edición. Editorial Mc Graw Hill Education. 
Bautista, J. (2019). Una caída de 34,4\% registro el aporte del turismo al PIB durante 2018 en Venezuela.

Bolivia emprende, (2018). Turismo generó más del mil millones de dólares y 294.000 empleos en 2018.

Boton, A. (2013). Analisis de casos de delitos forestale en el departamento del uiché. Universidad Rafael Landivar. Recuperado en http://biblio3.url.edu.gt/Tesario/2013/07/01/B oton-Ambany.pdf

Cámara Nacional del Trismo del Perú, (2018). WTTC: aporte total del turismo al Perú llega a US $\$ 20,838$ millones y $9.8 \%$ del PBI.

CEPAL, (2011). Impacto económico de las actividades mineras en la provincia de Jujuy. Recuperado en https://repositorio.cepal.org/bitstream/handle /11362/3875/1/S2011040.pdf

CEPAL, (2018). Estado de situación de la minería en América Latina y el Caribe: desafíos y oportunidades para un desarrollo más sostenible. Recuperado en

https://www.cepal.org/sites/default/files/pres entation/files/181116 extendidafinalconferen cia a los ministros mineria lima.pdf

Chadid, M. (2014). Patrones y dinámicas de deforestación en la serranía San Lucas, Colombia. Tesis de pregrado, Facultad de ciencias, Universidad Nacional de Colombia. Recuperado en http://bdigital.unal.edu.co/49018/1/Tesis\%20 San\%20lucas\%20final.pdf

El comercio, (2019). Ecuador reconoce que vive "Boom minero" y promete proteger el medioambiente. Recuperado en https://www.elcomercio.com/actualidad/ecua dor-boom-mineria-ambiente-economia.html

FAO y MAGBMA, (2016). Estudio De las causas de la deforestación y degradación forestal en Guinea ecuatorial. Recuperado en http://www.fao.org/3/CA0399ES/ca0399es.p $\underline{\mathrm{df}}$

FEDESARROLLO, (2008). La minería en

Colombia: impacto socioeconómico y fiscal. Recuperado en https://repository.fedesarrollo.org.co/bitstrea $\mathrm{m} /$ handle/11445/1012/Repor Abril \%20200 8 Cardenas y Reina.pdf?sequence=3\&isAll owed $=y$
García, V. (2008). El análisis de la deforestación en el bosque de protección altomayo mediante técnicas de Geomática. Tesis de maestría, Universidad de Piura. Repositorio Institucional Pirhua. Recuperada en https://pirhua.udep.edu.pe/bitstream/handle/ 11042/1476/MAS GAA 004.pdf?sequence= 1\&isAllowed =y

Guerra, F. (2016). Nivel de conocimiento de los estudiantes de educación secundaria del caserio Nina Rumi en relación a la conservación y mantenimiento de los bosques aledaños, Nina Rumi - San Juan Bautista - Manas - Loreto - Perú.

Gutiérrez, G. (2016). Programa de conservación y restauración en el área natural protegida parque nacional lagunas de Zempoala. Universidad Autónoma del Estado de México. Recuperado en http://ri.uaemex.mx/bitstream/handle/20.500. 11799/65757/tesis\%20axel\%20gtz.\%2004\% 20\%20octJGGC-splitmerge. pdf? sequence $=3$ \&isAllowed $=y$

IDEAM (2013). Boletín forestal 2011.

Informe de PIB, (2019). Brasil, el PIB cae respecto al trimestre anterior. Recuperado en https://datosmacro.expansion.com/pib/brasil

Jara, J. (2015). La deforestación de los bosques protectores como un atentado al derecho al buen vivir en la legislación ecuatoriana. Universidad Central del Ecuador.

Jiménez, H. (2015). En clave: la producción minera en Venezuela.

Juste, I. (2019). Causas de la deforestación. Ecología verde. Recuperado en https://www.ecologiaverde.com/causas-dela-deforestacion-258.html

Martínez, M. (2009). Reforestacion creación de cinturones verdes en el suelo de conservación ecológica del distrito Federal. Instituto tecnológico y de estudios superiores Monterrey. Tesis de Maestría, recuperado en

https://repositorio.tec.mx/bitstream/handle/11 285/569995/DocsTec 10886.pdf?sequence= 1\&isAllowed=y

Milán, A. (2017). Diseño del plan de reforestación en áreas estratégicas para el sistema de acueducto pertenecientes al municipio de Aguachica-Cesar. Universidad Francisco de Paula Santander. 
Ministerio de economía y finanzas Ecuador, (2018). USD 3800 millones de inversión minera hasta 2021 darán más prosperidad al Ecuador. Recuperado en https://www.finanzas.gob.ec/usd-3-800millones-de-inversion-minera-hasta-2021daran-mas-prosperidad-al-ecuador/

Ministerio de Minería, (2018). Aporte impositivo de la minería llego a \$US235 millones en 2017.

Ministerio de turismo, (2018). Redición de cuentas. Recuperado en

https://www.turismo.gob.ec/wpcontent/uploads/2019/02/Informe-Rendiciónde-Cuentas-2018-MINTUR.pdf

Miranda, L. (2013). Monitoreo de la deforestación mediante técnicas geomáticas en una porción de la región centro - norte de México. Universidad Autónoma de Nuevo León.

Mogrovejo, J. Herrera, V. Maldonado, L. García, E. (2019). El Turismo como contribución al crecimiento del PIB, para el desarrollo de la economía del país. Clío América, 13(25). Recuperado en https://revistas.unimagdalena.edu.co/index.p hp/clioamerica/article/view/3414

Monjardin, S. Pacheco, C. Plata, W. y Corrales, G. (2017). La deforestación y sus factores causales en el estado de Sinaloa - México. Madera y Bosques, vol. 23, núm. 1, 2017, pp. 7-22 Instituto de Ecología, A.C. Xalapa, México.

ONF Andina, (2018). Estudios de economía forestal en el marco de la misión de crecimiento verde en Colombia. Resumen ejecutivo. Gobierno de Colombia.

Recuperado en, https://www.dnp.gov.co/CrecimientoVerde/Documents/ejestematicos/forestal/Productos\%20finales/Res umen\%20V2018-09-30.pdf

Ortiz, J. Cancino, S. Cancino G. (2019). El aporte del turismo al desarrollo económico del municipio de Pamplona, región nororiental de Colombia.

Portafolio, Diario. (2019). La minería sigue siendo clave para la economía del gobierno.

Recuperado en https://www.portafolio.co/economia/lamineria-sigue-siendo-clave-para-la-gobierno$\underline{529442}$
Suqui, A. y Merecí, J. (2014). Efectos de la deforestación sobre las propiedades físicoquímicas de los suelos de la microcuenca del rio Zhurucay. Tesis de pregrado, Universidad de cuenca, Facultad de ciencias agropecuarias. Recuperado en http://dspace.ucuenca.edu.ec/bitstream/1234 56789/19978/1/TESIS.pdf

Torracchi, J. (2015). Deforestación y pérdida de hábitat en bosques de montaña en la cuenca alta del rio Zamora (Loja Ecuador). Universidad Politécnica Madrid.

UPRA, (2015). Zonificación para plantaciones forestales con fines comerciales - Colombia.

Urquiza, J. Burga, M. (2016). Incremento de la deforestación y sus consecuencias en la perdida de biomasa en los bosques de la región Loreto, 2000-2014. Universidad Nacional Autónoma del Perú.

Vera, G. (2015). RAISG: O Desmatamento na Amazônia desde 1970 até 2013RAISG: La Deforestación en la Amazonía desde 1970 hasta 2013RAISG: Deforestation in the Amazon from 1970 to 2013 